\title{
Collider detection of dark matter electromagnetic anapole moments
}

\author{
Alexandre Alves, ${ }^{1,2, *}$ A. C. O. Santos, ${ }^{2,3}$ and Kuver Sinha ${ }^{4, \dagger}$ \\ ${ }^{1}$ Departamento de Física, Universidade Federal de São Paulo, Diadema-SP 09972-270, Brazil \\ ${ }^{2}$ Departamento de Física, Universidade Federal da Paraíba, João Pessoa-PB, 58051-970, Brazil \\ ${ }^{3}$ Centre for Cosmology, Particle Physics and Phenomenology (CP3), Universit catholique de Louvain, \\ B-1348 Louvain-la-Neuve, Belgium \\ ${ }^{4}$ Department of Physics and Astronomy, University of Oklahoma, Norman, Oklahoma 73019, USA
}

(Received 10 November 2017; published 15 March 2018)

\begin{abstract}
Dark matter that interacts with the Standard Model by exchanging photons through higher multipole interactions occurs in a wide range of both strongly and weakly coupled hidden sector models. We study the collider detection prospects of these candidates, with a focus on Majorana dark matter that couples through the anapole moment. The study is conducted at the effective field theory level with the mono- $Z$ signature incorporating varying levels of systematic uncertainties at the high-luminosity LHC. The projected collider reach on the anapole moment is then compared to the reach coming from direct detection experiments like LZ. Finally, the analysis is applied to a weakly coupled completion with leptophilic dark matter.
\end{abstract}

DOI: 10.1103/PhysRevD.97.055023

\section{INTRODUCTION}

One of the defining features of dark matter (DM) models is the nature of the interaction between the DM candidate and Standard Model (SM) particles. This interaction is usually assumed to be mediated by heavy states (for example, the SM $Z$ or Higgs boson [1,2], or $Z^{\prime}$ bosons [3,4] belonging to extensions of the SM), although in recent times there has been a surge of interest in hidden-sector models in which the interaction is mediated by new dark forces [5].

A class of models with a long history that lies somewhat between these two options is one in which DM interacts with the SM by exchanging photons through higher multipole interactions [6-16]. At dimensions 5 and 6, the effective operators for multipole interactions of a DM fermion $\chi$ can be written as follows:

$\mathcal{L} \supset \frac{d_{M}}{2} \bar{\chi} \sigma^{\mu \nu} \chi F_{\mu \nu}+\frac{d_{E}}{2} \bar{\chi} \sigma^{\mu \nu} \gamma^{5} \chi F_{\mu \nu}+\mathcal{A} \bar{\chi} \gamma^{\mu} \gamma^{5} \chi \partial^{\nu} F_{\mu \nu}$.

Here, $d_{M}, d_{E}$, and $\mathcal{A}$ denote the magnetic, electric, and anapole moments, respectively. For Majorana DM, only the anapole operator is nonzero and can be written in effective field theory (EFT) as

\footnotetext{
aalves@unifesp.br

kuver.sinha@ou.edu
}

Published by the American Physical Society under the terms of the Creative Commons Attribution 4.0 International license. Further distribution of this work must maintain attribution to the author(s) and the published article's title, journal citation, and DOI. Funded by SCOAP ${ }^{3}$.

$$
\begin{aligned}
\mathcal{A} & \equiv \frac{g}{\Lambda^{2}}, \\
\mathcal{L}_{\text {eff,anapole }} & =\frac{g}{\Lambda^{2}} \bar{\chi} \gamma^{\mu} \gamma^{5} \chi \partial^{\nu} F_{\mu \nu},
\end{aligned}
$$

where $\Lambda$ is the cutoff scale.

These effective descriptions have been studied in a variety of UV completions-for example, models of technicolor [8,12], composite DM [13], supersymmetry [6], and, recently, simplified models of leptophilic DM $[17,18]$. In weakly coupled completions, DM is assumed to couple at tree level to heavy charged states and hence at one loop to the photon. Restricted to the case of supersymmetry, this could be a model of bino DM coupling to sleptons [19]. In strongly coupled completions, the DM candidate can be a composite state of charged particles. The cutoff scale $\Lambda$ corresponds to either the mass of heavy charged states running in the loop or the scale of confinement in the strongly coupled hidden sector.

The purpose of this paper is to explore the detection prospects at the high luminosity LHC (HL-LHC) of DM with couplings to the SM shown in Eq. (1.1). We focus, in particular, on the anapole operator of Eq. (1.2) and calculate the reach of the HL-LHC in probing the cutoff scale $\Lambda$ for different DM masses. A collider study of electric and magnetic dipole moments is left for future work. ${ }^{1}$ Our collider study is conducted in the conservative and comparatively clean mono- $Z$ channel, incorporating varying

\footnotetext{
${ }^{1}$ We note that collider studies of magnetic dipole DM have been performed in [20,21], for both the LHC and the ILC, using techniques different from the ones employed in our paper.
} 
levels of systematic uncertainties. We utilize analysis methods developed recently by a subset of the authors. After carefully choosing kinematic variables that can discriminate between signal and SM background in Sec. II, we select cuts using the Bayesian optimization method implemented in the Python algorithm HYPEROPT [22]. A boosted decision tree (BDT) is then used to classify events into signal and background classes, after a joint optimization of kinematic cuts and BDT hyperparameters.

We have two main motivations for this study. The first is to connect to the rather extensive body of literature on direct detection prospects for this class of DM models. Electromagnetic anapole and dipole DM has been investigated in the context of experiments like DAMA, CDMS, XENON, and LUX by several groups [10,23-30], and projections for the future LZ experiment based on a simple scaling of the scattering cross section have been given in $[17,18]$. At the level of the EFT, the reach in the cutoff scale $\Lambda$ obtained from our collider study can be mapped onto a reach in the value of the anapole moment $\mathcal{A}$ through Eq. (1.2) [taking dimensionless couplings $g \sim \mathcal{O}(1)$ ]. Since the anapole moment determines the scattering cross section of DM off nuclei, the collider reach can then be compared to the reach of DM direct detection experiments. We do this in Fig. 6, comparing the values of the anapole moment probed by LUX and LZ to the values probed by our collider study, assuming $5 \%, 10 \%$, and $20 \%$ systematic uncertainties at the HL-LHC with $3000 \mathrm{fb}^{-1}$ of data.

A second motivation for our paper lies in applying this comparative study to a particular UV completion. Over the past few years, there has been steady interest in models of leptophilic DM interacting with the SM via heavy charged mediators [31-35]. A comprehensive one-loop analysis of the direct detection phenomenology of this class of models has been performed by [34]. The relic density and indirect detection rates have been calculated by [34], and their embedding within supersymmetry has been studied by [31]. In [36], constraints on this class of models were obtained under the assumption of a DM spike near the supermassive black hole at the center of our Galaxy. For small mass separation between the charged mediators and the DM candidate, these models are difficult to probe at the LHC by direct production of the mediators themselves $[37,38]$. On the other hand, an explicit calculation reveals that the anapole moment is enhanced precisely in these compressed regions of parameter space. Since the coupling to the photon increases, we obtain a corresponding enhancement in the performance of our collider study, as well as the scattering cross section with nuclei. The expectation, then, is that our collider study, in conjunction with projections from LZ, should be able to probe these compressed parameter regions. This aspect of our study is conducted in Sec. VI.

The paper is structured as follows. After performing our collider analysis in Sec. II, the results of our EFT analysis are displayed in Fig. 5 in terms of the cutoff scale $\Lambda$, and in Fig. 6 in terms of the anapole moment $\mathcal{A}$. In the latter figure, the limits from current and future direct detection experiments are also displayed, following a discussion of the methods used to calculate those limits in Sec. V. The EFT results are then applied to the case of a simplified model with charged mediators in Sec. VI. We end with our conclusions.

\section{COLLIDER STUDY}

In this section, we first provide a brief overview of prior work on multipole DM. We then present the results of our collider study.

The relic density of anapole and dipole DM has been worked out by many authors [39-44]. In particular, the authors of [43] calculated the relic density in the DM mass range $m_{\chi} \sim \mathcal{O}(100-500) \mathrm{GeV}$, incorporating annihilation channels like $\chi \chi \rightarrow W^{+} W^{-}$and $\chi \chi \rightarrow t \bar{t}$. For DM with $m_{\chi} \sim 100 \mathrm{GeV}$, the correct thermal relic density is obtained for a value of the cutoff scale $\Lambda \sim 700 \mathrm{GeV}$. While noting, from our Fig. 5, that this critical value of $\Lambda$ will be probed at the HL-LHC, we will in general remain agnostic about the relic density constraint. Depending on the cosmological history of the Universe prior to big bang nucleosynthesis, a wide range of relic densities can in any case be allowed $[45,46]$.

Low mass multipole DM has been studied by several groups in the context of anomalies in direct detection experiments [10,23-30]. We note that low mass multipole DM has also found applications in addressing the longstanding solar abundance problem (discrepancies between solar spectroscopy and helioseismology) [47]. The preferred anapole moment in such models turns out to be $\Lambda \sim \mathcal{O}(1) \mathrm{GeV}$. However, in our current paper, we restrict our attention to DM with mass $\sim \mathcal{O}(100) \mathrm{GeV}$. Collider searches for low mass anapole or dipole DM will require different techniques that are left for future investigation.

We now turn to a discussion of the collider prospects of anapole DM with an emphasis on mono- $X$ channels, where $X=j, \gamma, Z, h[22,48-76]$. These channels can serve as fertile places to search for anapole DM at colliders. The only requirement is that initial or final states contain charged particles which can emit a photon, which will then ultimately couple to the anapole DM. To our knowledge, the earliest appraisal of anapole DM in the context of the LHC appeared in [43]. The authors performed a monojet study with $19.5(10.5) \mathrm{fb}^{-1}$ of CMS (ATLAS) data at $8 \mathrm{TeV}$ and obtained bounds on the cutoff scale $\Lambda 350 \mathrm{GeV}$. We note that the monojet cross section is expected to dominate over the mono- $Z$ signature for this class of models. Moreover, the mono- $Z$ channel suffers from the usual branching ratio penalty of demanding $Z$ decay to leptons. On the other hand, though, we also expect that systematic uncertainties on the background should scale more favorably for the mono- $Z$ process at the 
HL-LHC. A comparative study of mono- $Z$ and monojet signatures for this class of models at the HL-LHC is left for future work. For now, our collider results based on the mono- $Z$ signature should be treated as a conservative estimate.

The mono- $Z$ channel, moreover, offers a good compromise between the signal production cross section on the one hand and the information available in the $Z$ boson decay on the other. Our strategy will be to use the final state particle distributions to train a decision tree algorithm in order to efficiently separate signal and background events. While allowing $Z$ boson decays to jets would increase the number of signal events, the SM background associated with jets plus missing $E_{T}$ is large. On the other hand, the leptonic decay mode is a viable alternative since it is a cleaner channel and the associated cross section is not too much smaller. Previous searches for dark matter in the mono- $Z$ channel with and without machine learning tools showed good discovery prospects $[4,77]$.

We therefore perform a mono- $Z$ search in the leptonic channel at the $13 \mathrm{TeV}$ LHC. Our signal is

$$
p p \rightarrow Z+\gamma^{*} \rightarrow \ell^{+} \ell^{-}+\chi \bar{\chi},
$$

where $\ell=\mu, e$ come from the $Z$ boson and the dark matter pair from the virtual photon. The backgrounds considered in this work are the main irreducible ones

(i) $Z Z\left(\gamma^{*}\right) \rightarrow \ell^{+} \ell^{-}+\nu_{\ell} \bar{\nu}_{\ell}$, and

(ii) $W^{+} W^{-} \rightarrow \ell^{+} \ell^{\prime-}+\nu_{\ell} \bar{\nu}_{\ell^{\prime}}$,

and the main reducible ones

(i) $Z W \rightarrow \ell^{ \pm} \ell^{\mp} \ell^{ \pm}+\nu_{\ell^{\prime}}$, and

(ii) $t \bar{t} \rightarrow W^{+} W^{-} b \bar{b} \rightarrow \ell^{+} \ell^{\prime-}+\nu_{\ell} \bar{\nu}_{\ell^{\prime}}+j j$.

The irreducible $\tau^{+} \tau^{-}$background is very small after $\tau$ decays to lighter leptons. The single top background $W t$ has a final state similar to the $t \bar{t}$ background when the $W$ boson and the top quark decay leptonically but with a somewhat smaller jet multiplicity. Yet, just like $t \bar{t}$, as we are going to see in the next section, the larger jet multiplicity makes the $W t$ identification by the BDT classifier very efficient. Because the $W t$ rate is an order of magnitude smaller that $t \bar{t}$, it can safely be neglected.

We require the following basic selection criteria for the mono- $Z$ events:

$$
\begin{aligned}
p_{T}(\ell) & >20 \mathrm{GeV}, \quad\left|\eta_{\ell}\right|<2.5, \\
\Delta R_{\ell \ell} & >0.4, \quad E_{T}^{\prime}>20 \mathrm{GeV},
\end{aligned}
$$

two opposite-charge leptons (electrons or muons) with transverse momentum greater than $20 \mathrm{GeV}$ in the central region of the calorimeter, and missing energy larger than $20 \mathrm{GeV}$ for trigger purposes. These initial cuts are loose since ultimately we will tune the $\mathscr{E}_{T}$ cut concurrently with tuning the hyperparameters of the machine learning (ML) algorithm. This approach proved to be very efficient in optimizing the performance of the decision trees algorithm
TABLE I. Signal and main background cross sections after basic cuts of Eq. (2.2) in fb at the $13 \mathrm{TeV}$ LHC. The $W t$ background turns out to be negligible after the BDT classification.

\begin{tabular}{lccccc}
\hline \hline Signals & $100 \mathrm{GeV}$ & $200 \mathrm{GeV}$ & $300 \mathrm{GeV}$ & $400 \mathrm{GeV}$ & $500 \mathrm{GeV}$ \\
\hline$\sigma[\mathrm{fb}]$ & 0.143 & 0.119 & 0.095 & 0.073 & 0.056 \\
Backgrounds & $Z Z$ & $W W$ & $Z W$ & $t \bar{t}$ & $W t$ \\
$\sigma[\mathrm{fb}]$ & 152.4 & $1.5 \times 10^{3}$ & 236.2 & $1.4 \times 10^{4}$ & 584.9 \\
\hline \hline
\end{tabular}

in SM double Higgs production [57], a project undertaken recently by some of the authors.

We also do not demand an explicit jet veto at this point. Instead, we pass the number of reconstructed jets and leptons to the decision trees algorithm in order to facilitate the identification of the reducible backgrounds, as discussed in the next section. The lack of a lepton invariant mass cut may be noticeable as well. The task of selecting the events by cutting on kinematic variables is the job of the BDT and may be delegated entirely to the ML training phase. We actually found that the BDT performs better when we keep the preselection of events at a minimum.

The DM effective operator in Eq. (1.2) was implemented in FeynRules [58]. Events were generated with MADGRAPH5[59] at leading order with one extra jet. The hard and soft jet regimes were matched in the MLM scheme [60] at appropriate separation scales. Hadronization was performed with PyTHIA6 [61]. For the detector simulation and jet clustering we used DELPHES3.3 [62] and FASTJET [63] with the anti-kt algorithm. The luminosity was fixed at $3 \mathrm{ab}^{-1}$ projecting the results to the end of the experiment.

The matched cross sections of signal and background processes after the basic selection criteria are displayed in Table I for DM masses from 100 to $500 \mathrm{GeV}$ and $\Lambda=1 \mathrm{TeV}$ at the $13 \mathrm{TeV}$ LHC.

\section{KINEMATIC VARIABLES FOR BDT DISCRIMINATION}

In this section, we describe the kinematic variables used to represent our simulated data. Each event is represented by a real-valued vector composed of the following ten kinematic variables, inspired by the mono- $Z$ study performed in Ref. [4]:

(i) Missing energy $\mathscr{E}_{T}$. This variable is used for both cutting and BDT training. Events with heavier dark matter are characterized by harder $E_{T}$ spectrum.

(ii) The variable $M_{\ell \ell}$, which is the invariant mass of the lepton pairs. It is useful in distinguishing between leptons from $Z$ and $W$ decays.

(iii) The product $\mathscr{E}_{T} \times \cos \left(\Delta \phi\left(\vec{E}_{T}^{\text {miss }}, \vec{p}_{T}^{Z}\right)\right)$, where $\Delta \phi$ is the angle between the two-dimensional vector $\vec{E}_{T}^{\text {miss }}$ and the transverse momentum $\vec{p}_{T}^{Z}$ of the $Z$ boson candidate. This variable is a measure of axial- $\mathscr{E}_{T}$, 

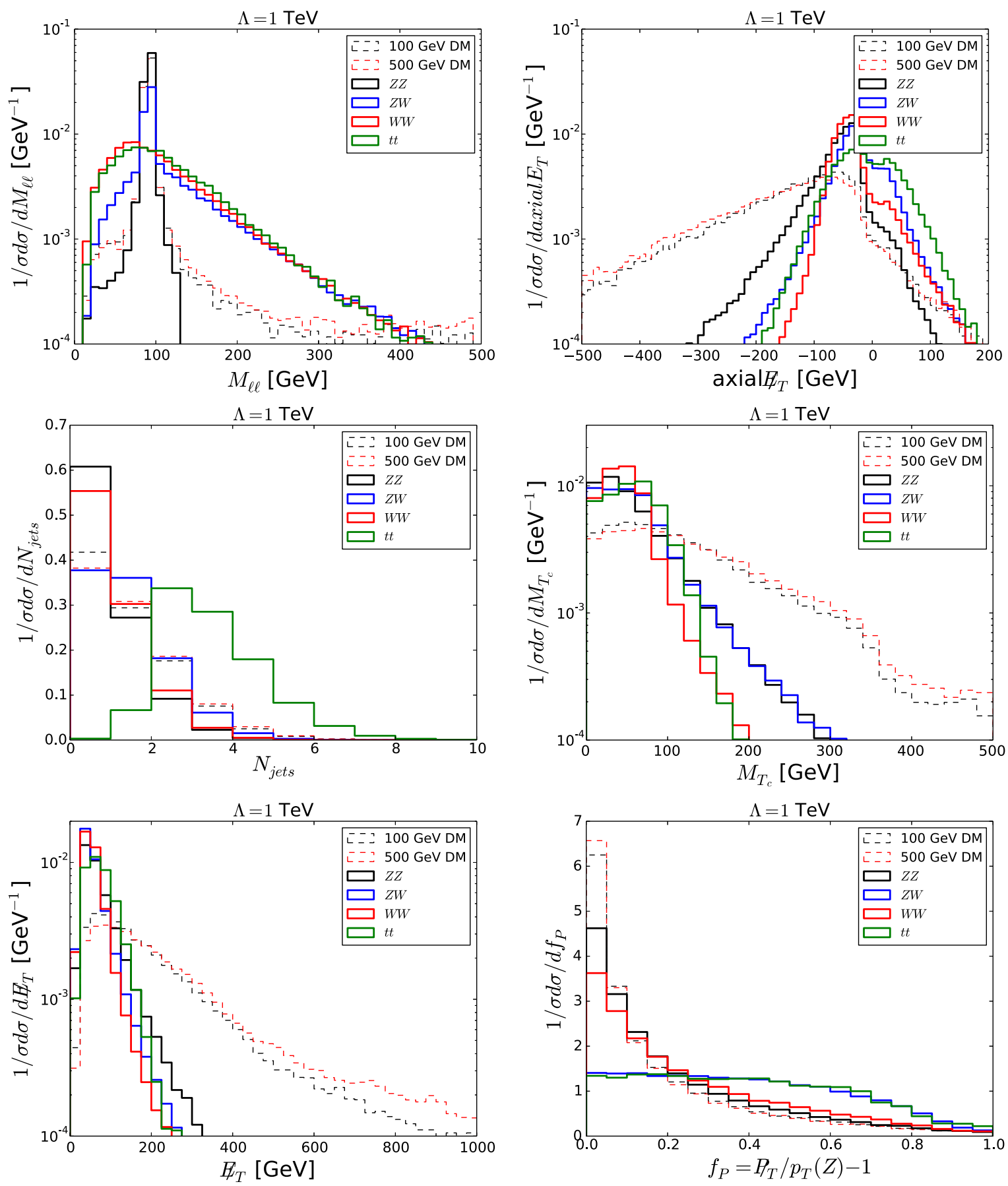

FIG. 1. Kinematic variables used in the BDT study. Upper panel: Plot of leptons invariant mass (left) and the axial- $E_{T}$ (right) distributions. Middle panel: Number of jets $N_{\text {jets }}$ (left) and the contransverse mass (right). Lower panel: Missing transverse energy distribution (left) and the fractional $p_{T}$ difference (right). The cutoff scale is fixed at $\Lambda=1 \mathrm{TeV}$. The solid lines correspond to the backgrounds and black (red) dashed lines correspond to DM distributions. The discriminative power of these variables decrease from upper to lower panels and from the left to right plots.

which is the projection of $\vec{E}_{T}^{\text {miss }}$ in the direction opposite to the $Z$ candidate [64]. It is useful to differentiate among various DM operators in EFT [77]. (iv) The variable $\left|E_{T}-p_{T}^{Z}\right| / p_{T}^{Z}$, which is the fractional $p_{T}$ difference [64].

(v) The variable $\Delta \phi\left(\ell^{+}, \ell^{-}\right)$, which is the azimuthal separation of the two leptons. 
(vi) The variable $\alpha_{T}=E_{T}\left(\ell_{2}\right) / M_{T}$, where $E_{T 2}$ is the transverse energy of the softest lepton of the $\ell^{+} \ell^{-}$pair and $M_{T}=\sqrt{\left(E_{T 1}+E_{T 2}\right)^{2}-\left(p_{x 1}+p_{x 2}\right)^{2}-\left(p_{y 1}+p_{y 2}\right)^{2}}$ [65].

(vii) The variable $\cos \left(\theta^{*}\right)$ [66] where $\theta^{*}$ is defined as the boost invariant $\cos \left(\theta^{*}\right)=\tanh \left(\frac{\eta_{\ell^{+}-}-\eta_{\ell^{-}}}{2}\right)$. This variable has been used in supersymmetric studies and is correlated to the production angle of sparticles. It has been studied in decay processes such as slepton to leptons $+E_{T}^{\prime}$ [66], or sbottoms to bottom jets + $E_{T}$ [67].

(viii) The variable $M_{T_{c}}=\sqrt{2\left(\vec{p}_{T_{\ell}} \cdot \vec{p}_{T_{\ell}}+p_{T_{\ell}} p_{T_{\ell}}\right)}$, which is the contransverse mass [68].

(ix) The variable $n_{j}$, which is the number of jets. Leptonic top quark pair production events present at least two hard jets.

(x) The variable $n_{\ell}$, which is the number of leptons identified in the event. The majority of $W Z$ events present three charged leptons.

Some of the variables defined above are shown in Fig. 1. In the left upper plot we show the leptons invariant mass $M_{\ell \ell}$ distribution for the backgrounds (solid lines) and for the lighter $(100 \mathrm{GeV})$ and the heavier $(500 \mathrm{GeV})$ dark matter considered in this work. A feature importance analysis demonstrated that this is the most discriminative variable in our multivariate analysis (MVA). Its importance is followed by the axial- $E_{T}$ variable (upper right), which combines the information of the missing transverse energy and the difference of the azimuthal angle of the two hardest charged leptons of the event, the number of jets (middle left), the contransverse mass (middle right) and the missing transverse energy (lower left). The least discriminative among the variables chosen to represent the data is the fractional $p_{T}$ difference displayed in the lower right panel of Fig. 1, yet removing it from the representation decreases the overall performance of the MVA. It is possible that a representation comprising more variables might enhance the power of the BDT to separate signals and backgrounds, but we found that these variables do a very good job in improving the signal significance compared to a cut-andcount analysis.

Some other features are worthy of comment. As expected, the heavier the DM particle is, the harder its spectrum is compared to the softer backgrounds. DM events present more collimated leptons from the $Z$ boson decay compared to the backgrounds. Two good variables to discern the reducible backgrounds $Z W$ and $t \bar{t}$ are the transverse momentum of the lepton pair, which is balanced by $\not p_{T}$ in events containing $Z$ bosons but less balanced for events containing $W$ 's; see the lower right panel of Fig. 1. Finally, in the left middle panel of Fig. 1 is displayed the number of hard jets identified in the event. Events with top quarks contain at least two hard jets and a considerably large fraction of events with higher jet multiplicity compared to the other processes. As observed in Ref. [4], the other variables not shown in Fig. 1 present good discrimination power as well. In contrast to Ref. [4], however, we use all these variables to represent our simulated data in the learning process of the BDT classifier.

\section{BDT CLASSIFICATION AND PERFORMANCE}

In this section, we discuss our decision tree analysis and give our results.

We utilized the XGBoosT package [69] to train boosted decision trees. Approximately 1.5 million events were generated, with around 300000 for each event classi.e., the signal class and the four background classes. One hundred thousand events were singled out for optimization purposes and the remaining to train/validate and test the BDT in the proportion of $2 / 3$ and $1 / 3$ of the events, respectively.

Since the DM mass is not known at the stage when the mono- $Z$ signal is studied, it is not possible, in principle, to optimize the ML algorithm to discern the signal for a given mass. Yet, the DM mass in the signal class needs to be fixed to train the BDT. We chose to fix the DM mass at $100 \mathrm{GeV}$, the initial value of our mass scan. This choice was motivated by the fact that a $100 \mathrm{GeV}$ particle signal is harder to discern from the background than a heavier one, as can be observed from the distributions shown in Fig. 1. Our expectation is that the algorithm also presents a good performance for the heavier (and easier) dark matter signals. We checked that, in fact, heavier dark matter is more easily identified as a signal event in this approach. A more sophisticated approach, based on parametrized neural networks, is also possible [70].

The BDT hyperparameters, the $E_{T}$ threshold cut, and the number of leptons and jets in order a given event be vetoed were all adjusted jointly in a Bayesian optimization framework with HYPEROPT [22]. The joint optimization of cuts and ML hyperparameters is advantageous once the kinematic cuts affect the performance of these algorithms in a way that is hard to predict. Reducing the number of background events with hard cuts helps to increase the signal significance, but has a deleterious effect on the BDT classification as the kinematic distributions of the various classes become more similar to each other. Delegating all the discrimination to the ML side, on the one hand, might not suffice if the backgrounds are too much larger than the signal. The optimum point in this trade-off is achieved by the joint optimization as described in detail in Ref. [57].

The signal significance was calculated taking a systematic uncertainty in the total background yield, $\varepsilon_{B}$. We estimated four scenarios: one with almost no systematic uncertainties, taking $\varepsilon_{B}=1 \%$, and several others with varying degrees of uncertainties, taking $\varepsilon_{B}=5 \%, 10 \%$, and $20 \%$. The joint optimization described in the previous paragraph was performed taking these systematic uncertainties into account, in 


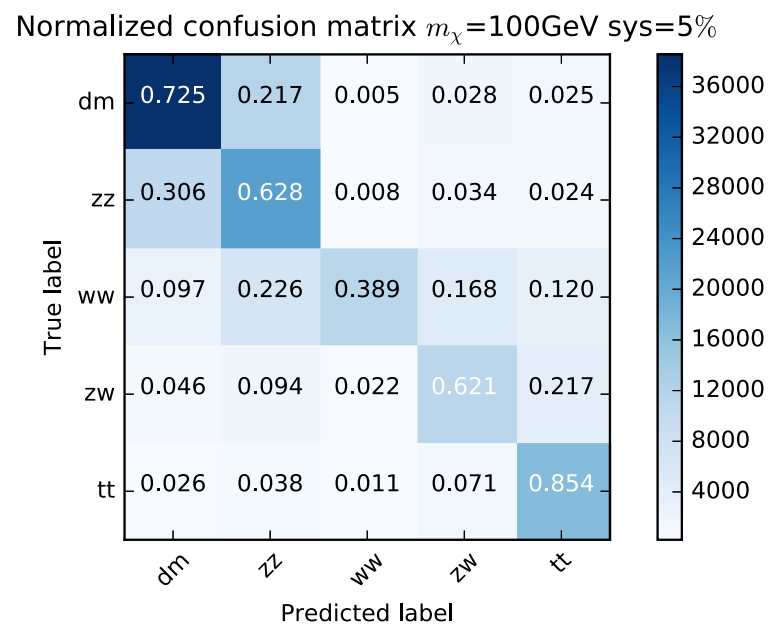

FIG. 2. BDT performance plot: Normalized confusion matrix for the multichannel analysis at $m_{\chi}=100 \mathrm{GeV}$, and systematic uncertainties of $5 \%$ for the test samples. The grid consists of the signal class (labeled "dm"), and the four major background classes (labeled $Z Z, Z W, W W$, and $t t$ ) corresponding to the backgrounds discussed below Eq. (2.1). It is evident that around $22 \%$ of the signal events are predicted to be $Z Z$ events and $30 \%$ of the $Z Z$ events are predicted to be signal events.

order that the optimization algorithm learns to increase the $S / B$ ratio and tame the effects of these uncertainties as explained in detail in Ref. [57].

We now show the performance plots for our BDT analysis. We will discuss, in turn, the confusion matrix, the receiver operating characteristic (ROC) curves, the BDT output distributions for signal and backgrounds, and, finally, the $\Lambda$ scale that can be probed with $3 \mathrm{ab}^{-1}$ of data at the $13 \mathrm{TeV}$ LHC, all obtained for the test samples.

In Fig. 2, we show the normalized confusion matrix for the multichannel analysis at $m_{\chi}=100 \mathrm{GeV}$, and systematic uncertainties of 5\%. The grid consists of the signal class (labeled "dm"), and the four major background classes (labeled $Z Z, Z W, W W$, and $t t$ ) corresponding to the backgrounds discussed below Eq. (2.1). As expected, it is the $Z Z$ background that is most difficult to discern from the signal. From the confusion matrix we see that around $22 \%$ of the signal events are predicted to be $Z Z$ events and $30 \%$ of $Z Z$ events are predicted to be signal events. The reducible $Z W$ and $t \bar{t}$ are easily identified as background events due to their larger lepton and jet multiplicities, respectively, as anticipated in the previous section.

In Fig. 3, we show the ROC curves for the multichannel analysis at $m_{\chi}=100 \mathrm{GeV}$, and systematic uncertainties of $5 \%$. The ROC translates to a curve whose points represent the background rejection for a given signal efficiency cutting on the BDT output. The larger the area below the ROC curve, the easier it is to separate signals from backgrounds based on a cut on the algorithm output scores associated with each event.

This can more easily be understood by considering the impact of the BDT cut on the signal significance. Let us call

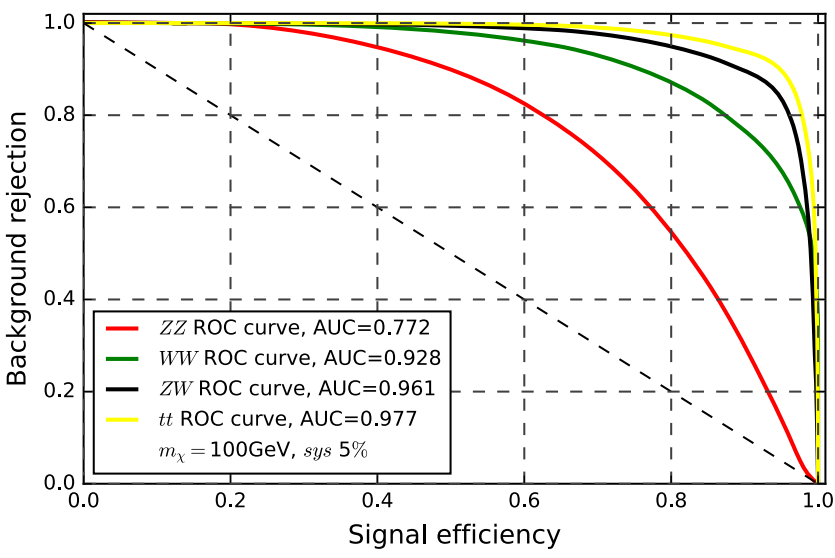

FIG. 3. BDT performance plot: Receiver operating characteristic (ROC) curves for the multichannel analysis at $m_{\chi}=100 \mathrm{GeV}$, and systematic uncertainties of $5 \%$ for the test samples. The red, green, blue, and yellow curves show the $Z Z$, $W W, Z W$, and $t \bar{t}$ backgrounds, respectively. The $Z Z$ background shows the lowest area under curve (AUC). The $t \bar{t}$ has a larger background rejection rate due its larger jet multiplicity.

$\epsilon_{S}^{\mathrm{BDT}}$ the fraction of signal events $N_{S}$ accepted after cutting on the BDT output of the events classified as signal in the figure shown below, and $r_{B}^{\mathrm{BDT}}$ the fraction of the background events $N_{B}$ rejected after the same BDT cut, and then

$$
\frac{\epsilon_{S}^{\mathrm{BDT}} N_{S}}{\sqrt{\left(1-r_{B}^{\mathrm{BDT}}\right) N_{B}}}
$$

is roughly the statistical significance of the signal. What we actually want is an $\epsilon_{S}^{\mathrm{BDT}}$ as close to 1 as possible and a $r_{B}^{\mathrm{BDT}}$ as close to 1 as possible. A perfect algorithm would produce a rectangular ROC whose maximum performance point would be the right upper corner of the rectangle. The better is the discrimination, the closer to 1 is the area under ROC; this is called the area under curve (AUC) in the ML literature, and it is displayed separately in the legend of Fig. 3 for each background.

The red, green, blue, and yellow curves of Fig. 3 show the $Z Z, W W, Z W$, and $t \bar{t}$ backgrounds, respectively. The results of the confusion matrix are corroborated by the ROC curves of Fig. 3. Indeed, it is the ZZ background that shows the smallest AUC, signifying the smallest background rejection for a given signal acceptance. We note, on the other hand, that $t \bar{t}$ has a larger background rejection rate, mainly because of its larger jet multiplicity. The lepton multiplicity helps to discern the $Z W$ backgrounds which has the second largest AUC. We found that the optimum $\mathscr{E}_{T}$ cut varies between 50 to $90 \mathrm{GeV}$ depending on the systematics level and no jet or lepton vetoes. That is, the best performance was achieved by delegating the task of enhancing the classification performance more to the BDT and less to the kinematical cuts.

We have also performed a feature importance analysis of the kinematic variables used in our work. The most 


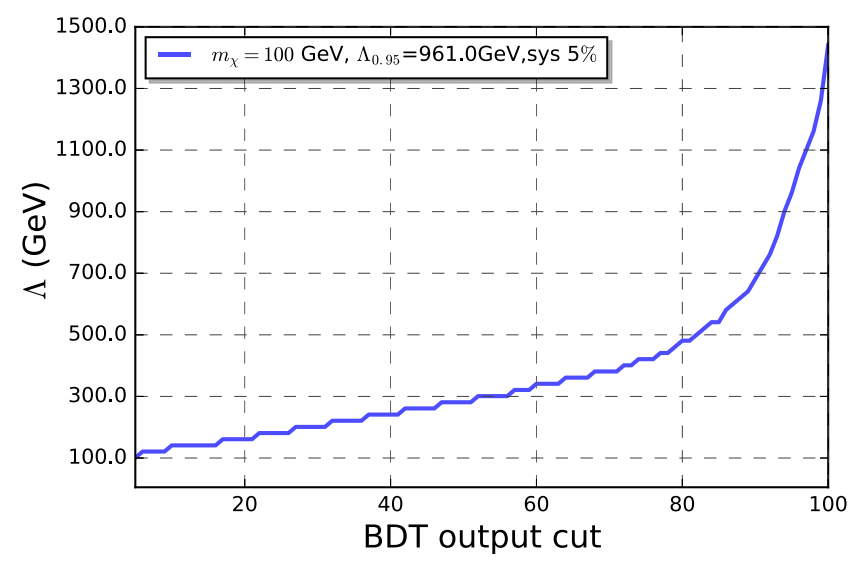

FIG. 4. BDT performance plot: The cutoff energy scale $\Lambda$ for discovery $(5 \sigma)$ as a function of BDT score cut for a $100 \mathrm{GeV}$ dark matter and assuming a 5\% systematics. We chose a cut of 0.95 in the BDT output to separate signal and backgrounds.

discriminative variable is the leptons invariant mass which is also the most important variable in cut-and-count analysis. This is followed by the axial- $E_{T}$ variable, the number of jets in the event, the contransverse mass, and the missing transverse momentum. A less discriminative variable is the fractional transverse momentum. In spite of the fact that the lepton invariant mass is the most powerful variable, the other variables are necessary to maintain the functionality and performance of the algorithm.

In Fig. 4 , we plot the cutoff energy scale $\Lambda$ for discovery $(5 \sigma)$ as a function of BDT score cut. We see that the $\Lambda$ scale which can be probed by the $13 \mathrm{TeV}$ LHC increases very rapidly as the output cut approaches 1 . We chose to keep the cut at 0.95 for the sake of the stability of the results. A harder cut probes regions with too few background events, which leads to larger fluctuations. In order to estimate the reach in $\Lambda$, we averaged the results of ten runs, randomly reshuffling the train and test samples at each run. The uncertainty of the LHC reach in $\Lambda$ is around $\pm 10 \%-15 \%$ of the estimated $\Lambda$ depending on the mass at the 0.95 output threshold. The results shown in Fig. 4 are from this averaging process, keeping a 5\% systematics level. Results for other DM masses and systematic errors were obtained in the same way. After the BDT output cut, 31(18) signal and 136(164) background events survive for a 100 (500) $\mathrm{GeV}$ dark matter.

In Fig. 5, we display the $5 \sigma$ reach in the cutoff energy scale $\Lambda$ as a function of DM mass for several values of systematics uncertainties. The luminosity is fixed at $3000 \mathrm{fb}^{-1}$. The performance of the BDT classifier improves as the dark matter gets heavier. This behavior can be understood when we look at the distributions of Fig. 1. For example, the $500 \mathrm{GeV}$ DM presents kinematic distributions which are less similar to the backgrounds. Most importantly, the signal distribution in that case is distinct from the $Z Z$ background, making the BDT

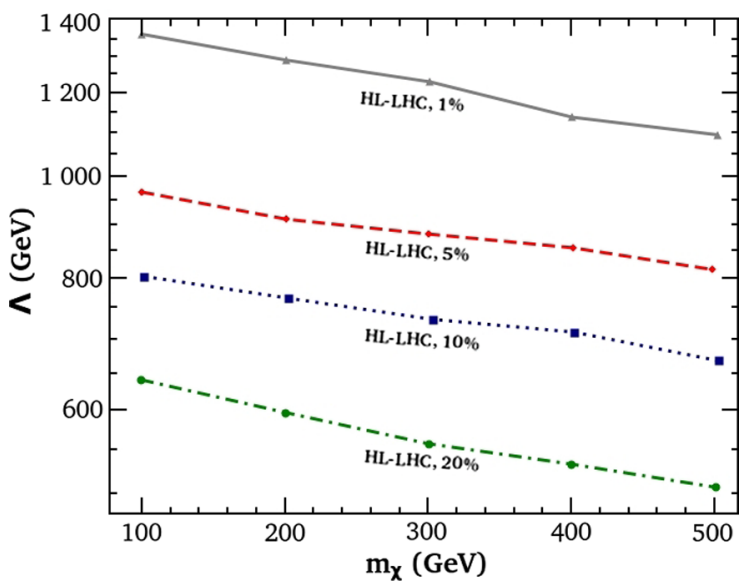

FIG. 5. Collider results: The $5 \sigma$ reach in the cutoff energy scale $\Lambda$ as a function of dark matter mass for several values of systematics uncertainties. The luminosity is fixed at $3000 \mathrm{fb}^{-1}$.

classification more efficient. Of course, as the cross section decreases with the DM mass, the $\Lambda$ scale that can actually be probed drops with the DM mass as shown in Fig. 5. In the case of the $300 \mathrm{GeV}$ mass, the reach of the scale $\Lambda$ changed only slightly compared to the $200 \mathrm{GeV}$ case-a persistent effect up to the $10 \%$ systematics level. In this case, the gain in the BDT classification is competitive with the drop in cross section. For larger masses, however, the number of signal events produced was not enough to beat the better classification achieved with the ML algorithm, and the estimated LHC sensitivity in $\Lambda$ is degraded.

Assuming a very small systematic uncertainty of $1 \%$, the scales for which the anapole DM can be discovered, at $5 \sigma$, are all above $\Lambda \sim 1.1 \mathrm{TeV}$, as can be seen in Fig. 5 . Assuming systematic uncertainties at the level of $5 \%$, a $100 \mathrm{GeV} \mathrm{DM}$ can still be discovered if $\Lambda \approx 1 \mathrm{TeV}$. From $5 \%$ to $10 \%$ and from $10 \%$ to $20 \%$ systematics, the discovery reach in $\Lambda$ decreases approximately by $200 \mathrm{GeV}$ for a given DM mass.

Before proceeding, we would like to contrast our mono$Z$ study with monojet studies. In the most recent ATLAS monojet study [79], which presents results for $36 \mathrm{fb}^{-1}$ of data combined from 2015 and 2016, the L1 and high level trigger (HLT) systems select events with missing energy above $90 \mathrm{GeV}$. After analysis selections, the trigger was measured to be fully efficient for events with $E_{T}>250 \mathrm{GeV}$. While in principle one can reduce the offline $E_{T}$ threshold during analysis while keeping the L1 and HLT triggers fixed, this is not advisable. One then suffers from loss of trigger efficiencies. Indeed, from Fig. 41(a) of [80], it is clear that for off-line $\not_{T}$ cuts below around $200 \mathrm{GeV}$, the trigger efficiency drops precipitously.

This is one of the reasons we have opted for a mono- $Z$ study with dilepton final states, where one does not have to rely on a hard $E_{T}$ trigger at the L1 and HLT stages, and can instead trigger on the two leptons. One can then apply an 
optimized off-line $E_{T}$ cut as dictated by the needs of the analysis, without suffering from loss of trigger efficiencies. In fact, BDT studies generally rely on lower $\mathscr{E}_{T}$ cuts, and it is possible that channels in which one can trigger on other states (such as dileptons) instead of relying on hard $E_{T}$ triggers may have a better performance, despite suffering from a lower cross section. A dedicated study of mono- $Z$ versus monojet in this class of models is left for the future.

\section{COMPARISON WITH DIRECT DETECTION LIMITS}

In this section, we compare the collider constraints on the anapole moment to constraints coming from direct detection experiments.

We are interested in DM masses $\mathcal{O}(100 \mathrm{GeV})$ and typical nuclear recoil energy $\sim 10-30 \mathrm{keV}$. This corresponds to a DM-nucleus momentum transfer

$$
\sqrt{\left|q^{2}\right|} \approx|\boldsymbol{q}| \approx 50 \sim 80 \mathrm{MeV}
$$

where $\boldsymbol{q}$ is the three-vector of the four-momentum $q$. We assume that the DM has a coupling with the electromagnetic field given by Eq. (1.2). At small momentum transfer, the interaction of the DM and the nucleus can be described by the following effective Lagrangian:

$$
\mathcal{L}_{\text {DM-nucleus }}=\frac{i \mathcal{A}}{2} \bar{\chi} \gamma^{\mu} \gamma^{5} \chi \partial^{\nu} F_{\mu \nu}+e A_{\mu} J^{\mu},
$$

where $J^{\mu}$ is the nuclear current operator. We are neglecting the $q^{2}$ dependence in $\mathcal{A}$; a detailed calculation performed in a previous paper by a subset of the authors revealed that this introduces at most a $0.6 \%$ error in the anapole moment for the energies considered here [18].

The differential cross section for the scattering of DM with nuclei is given by $[17,23,30,71]$

$$
\begin{aligned}
\frac{d \sigma}{d E_{R}}= & 4 \alpha_{\mathrm{em}} \mathcal{A}^{2} Z^{2} F_{Z}^{2}\left(\boldsymbol{q}^{2}\right)\left[2 m_{T}-\left(1+\frac{m_{T}}{m_{\chi}}\right)^{2} \frac{E_{R}}{v^{2}}\right] \\
& +4 \mathcal{A}^{2} d_{A}^{2} F_{s}^{2}\left(\frac{J+1}{3 J}\right) \frac{2 E_{R} m_{T}^{2}}{\pi v^{2}} .
\end{aligned}
$$

Here, the mass of the target nucleus is denoted by $m_{T}$, $E_{R}=\boldsymbol{q}^{2} /\left(2 m_{T}\right)$ denotes the recoil energy of the nucleus, $Z$ is the nuclear charge, and $v$ is the velocity of the DM particle. $F_{Z}$ is the nuclear charge form factor, while the nuclear spin form factor is denoted by $F_{s}$. The second term corresponds to scattering off the nuclear dipole moment $d_{A}$, which is small for xenon.

The differential rate per unit target mass is

$$
\frac{d R}{d E_{R}}=\frac{\rho_{0}}{m_{\chi} m_{T}} \int_{v_{\min }}^{\infty} d^{3} v \frac{d \sigma}{d E_{R}} v f_{\oplus}(\vec{v}),
$$

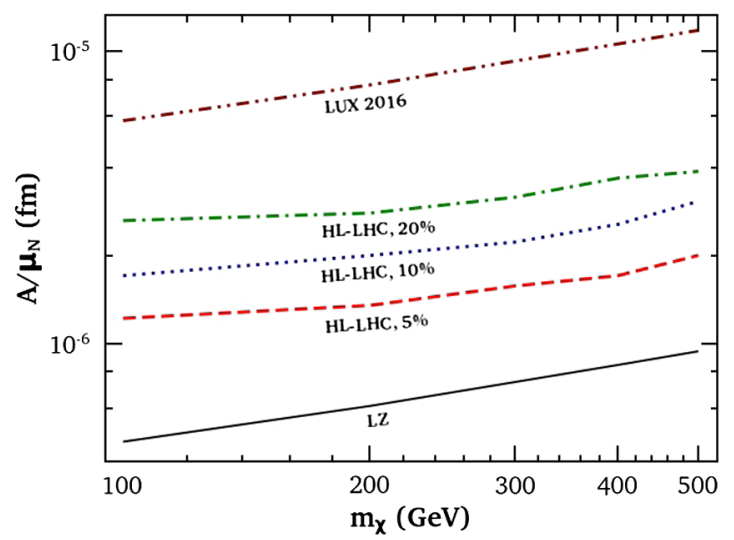

FIG. 6. A comparison between the bounds coming from LUX 2016 [72] and projected LZ limits on the anapole moment versus those coming from our HL-LHC study with varying levels of systematic uncertainties. The $\mathrm{LZ}$ projection corresponds to the 1 event contour in Fig. 12.3.6 of [78].

where $\rho_{0}$ is the local DM density. The minimal velocity of DM that is required for a recoil energy $E_{R}$ is given by $v_{\text {min }}=\sqrt{m_{T} E_{R} / 2} / M_{\text {red }}$, where $M_{\text {red }}$ is the reduced mass of the DM-nucleon system. The DM velocity distribution in the rest frame of the detector is given by $f_{\oplus}(\vec{v})$.

Based on these calculations, several groups have calculated the scattering cross section of multipole DM. The constraints can be depicted as upper bounds on the anapole or dipole moments, and we will mainly use the results obtained in the $m_{\chi} \sim \mathcal{O}(100) \mathrm{GeV}$ range. The constraint on the scattering cross section for a given DM mass obtained from LUX 2016 [72] can be scaled to the corresponding projected constraint at LZ. We will take the most optimistic scenario, with one background event in 1000 days of exposure of 5.6 tonne fiducial mass [73]. This corresponds to the "1 event" contour in Fig. 12.3.6 of [78]. The exclusion limit on the scattering cross section for this optimistic projection of LZ is expected to be lowered by a factor of $\sim 7 \times 10^{-4}$ compared to the LUX 2013 results [74]. Clearly, this is a rough estimate, and a careful analysis of future data sets will be needed.

In this context, we note that LZ projections for anapole DM have also been performed in [44]. ${ }^{2}$ Our LZ limits are approximately an order of magnitude more stringent. Indeed, the $3 \sigma$ and $5 \sigma$ projection contours in Fig. 12.3.6 of [78] are far more conservative. For example, for DM with mass $\sim \mathcal{O}(100) \mathrm{GeV}$, we obtain $\mathcal{A} / \mu_{N} \sim 4 \times 10^{-7} \mathrm{fm}$, where $\mu_{N}$ is the nuclear magneton. On the other hand, the authors of [44] adopt the projection limit of $\mathcal{A} / \mu_{N} \sim 1 \times 10^{-6} \mathrm{fm}$. Clearly, using more conservative LZ projections would only

\footnotetext{
${ }^{2}$ The paper [44] studied anapole DM in the context of radiative seesaw models. The parameter space of interest in these models requires values of the anapole moment that are beyond the HL-LHC and LZ projections computed in our paper.
} 


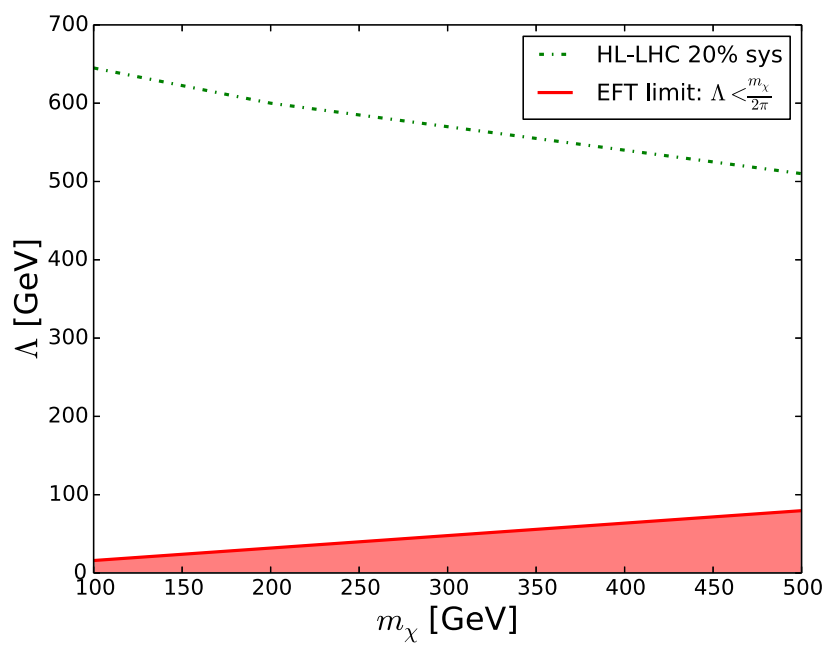

FIG. 7. EFT validity regime: The shaded region shows where the effective theory breaks down assuming a simple UV completion with a mediator in the $s$-channel.

increase the relative importance of our HL-LHC study vis-àvis direct detection.

The resulting comparative study between the collider reach and the reach from direct detection experiments is shown in Fig. 6. On the horizontal axis, we plot the DM mass in the range 100-500 GeV, while on the vertical axis we plot the anapole moment in units of the nuclear magneton. The LUX 2016 results are shown in the brown dash-dot-dotted curve, while the black solid curve shows the projected LZ limit. The green dot-dashed, blue dotted, and red dashed curves show the results obtained from our collider study, with $20 \%, 10 \%$, and $5 \%$ systematic uncertainties and $3000 \mathrm{fb}^{-1}$ of data at the HL-LHC, respectively.

We can see that there is a vast improvement, almost by an order of magnitude, in the most optimistic projected LZ limit compared to the current LUX limit. This corresponds to the fact that the constraint on the scattering cross section is expected to become stronger by $\sim 100$, although, as we have stressed, this is a rough estimate. On the other hand, the HL-LHC is expected to constrain the anapole moment by a factor of 2-6 compared to the current LUX results, depending on the level of systematics. It is possible that a study in other channels such as monojet, or a combination of channels, will strengthen the collider results.

Since the next section will place our study in the context of a UV completion, we make a few comments about the regime of validity of our effective approach. As discussed, for example, in [76], for the $s$-channel exchange of a mediator particle, the effective description is valid only for $m_{\text {med }} \geq 2 m_{\chi}$, where $m_{\text {med }}$ denotes the mediator mass. The cutoff scale is related to the mediator mass by $\Lambda \sim m_{\text {med }} / \sqrt{g_{1} g_{2}}$, where $g_{1,2}$ are Yukawa couplings, which are bounded by $g_{1} g_{2} \leq(4 \pi)^{2}$ from perturbativity requirements. This therefore gives a final regime where the effective description is valid: $\Lambda \geq m_{\chi} / 2 \pi$. This means that the entire region in Fig. 5 of our manuscript falls in the region where the effective description is valid; indeed, the invalid region is relegated to regions $\Lambda<100 \mathrm{GeV}$, far below our region of interest. ${ }^{3}$

We display the region where the effective theory is valid in Fig. 7 and add a small caveat regarding its interpretation. Namely, our UV completion in Sec. VI is not a model with a simple $s$-channel exchange, so this particular demarcation is not strictly valid for the rest of the paper, although it is the simplest general illustration of the fact that the effective collider analysis performed in the paper is valid for a large class of the simplest $s$-channel completions.

\section{APPLICATION TO A SIMPLIFIED MODEL}

In this section, we finally apply our EFT analysis to a specific simplified model. We choose a weakly coupled UV completion in which the DM is a Majorana fermion $\chi$ that couples to an uncolored fermion $f$ (with mass $m_{f}$ ) and a pair of charged scalars $\tilde{f}_{L, R}$. At one loop, the DM couples to the photon through an anapole moment interaction. The mass of the charged scalars will be taken to be $\sim 250 \mathrm{GeV}$, while the results taken from the EFT will correspond to a cutoff scale $\Lambda \sim 800 \mathrm{GeV}$. While the EFT results can be trusted to a first approximation, we note that a detailed collider study of the simplified model will yield more precise constraints.

The Lagrangian of the model is given by

$$
\mathcal{L}_{\text {int }}=\lambda_{L} \tilde{f}_{L}^{*} \bar{\chi} P_{L} f+\lambda_{R} \tilde{f}_{R}^{*} \bar{\chi} P_{R} f+\text { c.c. }
$$

A nonzero mixing angle $\alpha$ is allowed between the scalar mass and chiral eigenstates

$$
\left(\begin{array}{l}
\tilde{f}_{1} \\
\tilde{f}_{2}
\end{array}\right)=\left(\begin{array}{cc}
\cos \alpha & -\sin \alpha \\
\sin \alpha & \cos \alpha
\end{array}\right)\left(\begin{array}{l}
\tilde{f}_{L} \\
\tilde{f}_{R}
\end{array}\right) .
$$

The two scalar mass eigenvalues are denoted by $m_{\tilde{f}_{1}}$ and $m_{\tilde{f}_{2}}$. The free parameters of the model are the four masses $m_{\chi}, m_{\tilde{f}_{1}}, m_{\tilde{f}_{2}}$, and $m_{f}$.

A supersymmetric embedding of this model has been studied in $[31,81]$. Here, we briefly summarize the dependence of the anapole moment on the model parameters. For a full derivation, we refer to the appendix of [18].

The relevant Feynman diagrams consist of a triangle loop with either two fermions $f$ or two scalars $\tilde{f}$, and external legs given by two DM particles and a photon.

\footnotetext{
${ }^{3}$ For monojet studies, the truncation between the validity of the effective description and the regime where the details of the underlying simplified model become important were studied in [75]. This study found that the effective description can be trusted for mediator masses $m_{\mathrm{med}}^{2} \geq m_{\mathrm{DM}}^{2}+E_{T}^{2}$. This is not very surprising, since the underlying assumptions are still (i) $s$-channel mediator exchange and (ii) perturbativity of Yukawa couplings.
} 
Let us take the momentum of the incoming and outgoing DM particles to be given by $p$ and $p^{\prime}$, respectively. The total off-shell scattering amplitude is given by

$$
\mathcal{M}^{\mu}=i \mathcal{A}\left(q^{2}\right) \bar{u}\left(p^{\prime}\right)\left(q^{2} \gamma^{\mu}-\not q q^{\mu}\right) \gamma^{5} u(p),
$$

where the momentum transfer is denoted by $q=p^{\prime}-p$ and the anapole moment by $\mathcal{A}\left(q^{2}\right)$. The anapole moment $\mathcal{A}\left(q^{2}\right)$ can be expressed as

$$
\begin{aligned}
\mathcal{A}\left(q^{2}\right)= & e\left(\left|\lambda_{L}\right|^{2} \cos ^{2} \alpha-\left|\lambda_{R}\right|^{2} \sin ^{2} \alpha\right) X_{1}\left(q^{2}\right) \\
& +e\left(\left|\lambda_{L}\right|^{2} \sin ^{2} \alpha-\left|\lambda_{R}\right|^{2} \cos ^{2} \alpha\right) X_{2}\left(q^{2}\right),
\end{aligned}
$$

where $X_{1,2}$ is the result of three-point loop integrals. The derivation of these equations, along with the full form of $X_{i}$, is given in [18].

In the limit $\left|q^{2}\right| \ll m_{f}^{2}$ and $\left|q^{2}\right| \ll m_{\tilde{f}_{i}}^{2}$, the $X_{i}$ reduce to a simple expression,

$$
\begin{aligned}
X_{i} & {\left[q^{2}=0\right] \rightarrow \frac{1}{96 \pi^{2} m_{\chi}^{2}}\left[\frac{3 \mu_{i}-3 \delta+1}{\sqrt{\Delta_{i}}} \operatorname{arctanh}\left(\frac{\sqrt{\Delta_{i}}}{\mu_{i}+\delta-1}\right)\right.} \\
& \left.-\frac{3}{2} \log \left(\frac{\mu_{i}}{\delta}\right)\right],
\end{aligned}
$$

where $\Delta_{i}=\left(\mu_{i}-\delta-1\right)^{2}-4 \delta, \delta=m_{f}^{2} / m_{\chi}^{2}$, and $\mu_{1}=\frac{m_{f_{1}}^{2}}{m_{\chi}^{2}}$, $\mu_{2}=\frac{m_{\tilde{f}_{2}}^{2}}{m_{\chi}^{2}}, \delta=\frac{m_{f}^{2}}{m_{\chi}^{2}}$. This limit applies to DM direct detection for $f=\mu, \tau$. For very heavy mediators $\mu_{i} \gg 1, X_{i}$ vanishes as $\mu_{i}^{-1} \log \mu_{i}$. If the mass difference between $\tilde{f}$ and the DM is small, the value of $X_{i}$ will increase; in the limit $\left(\mu_{i}-1\right) \sim \delta \ll 1$,

$$
X_{i} \sim \frac{1}{96 \pi^{2} m_{\chi}^{2}}\left[\frac{\pi}{\sqrt{\delta}}-\frac{3}{2} \log \frac{1}{\delta}\right] .
$$

For $f=\mu$ and $\tau$, this model has a sizable anapole moment, which can be detected in direct detection experiments.

We now present the limits from various experiments shown in Fig. 6 in the parameter space of this class of models. But before doing so, we comment on the validity of using the collider analysis, which was performed in the effective limit, to our simplified model. Mapping between the effective theory and the simplified model, one obtains

$$
\begin{aligned}
& \Lambda^{2} \sim 96 \pi m_{\tilde{f}_{1}}^{2}, \\
& \quad g \sim \mu_{1} / \sqrt{\delta} \sim \mathcal{O}(1) .
\end{aligned}
$$

The large loop suppression renders the effective approximation robust in this case, although a comparative collider study between the effective theory and the simplified model would be interesting and is kept for future work. ${ }^{4}$

\footnotetext{
${ }^{4}$ The only such study in the mono- $Z$ channel, as far as we know, was performed by two of the current authors in [4].
}

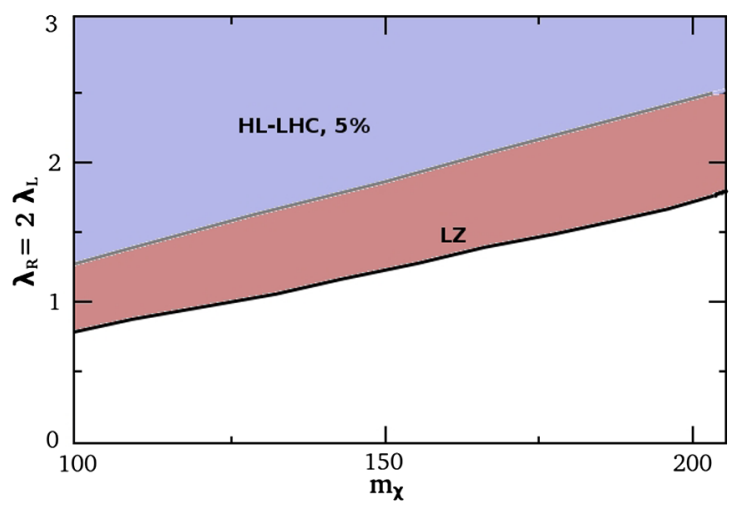

FIG. 8. Constraints on the $\left(\lambda, m_{\chi}\right)$ plane: We show the constraints on the $\lambda_{R}=2 \lambda_{L}$ versus the DM mass $m_{\chi}$ plane, keeping $\alpha=\pi / 4$ and $\mu_{1}=m_{\tilde{f}_{1}}^{2} / m_{\chi}^{2}=1.44$. The bluish grey region shows the part of parameter space that will be constrained by mono- $Z$ searches at the HL-LHC, assuming $3000 \mathrm{fb}^{-1}$ of data and $5 \%$ systematic uncertainties. The red region is the region that will be constrained by LZ, assuming the most optimistic performance with one background event in 1000 days of exposure of 5.6 tonne fiducial mass. The LZ projection corresponds to the 1 event contour in Fig. 12.3.6 of [78]. Current LUX limits and direct collider searches for the mediators $\tilde{f}_{1}$ are not able to constrain this part of parameter space.

In Fig. 8, we first plot the constraints on the $\left(\lambda, m_{\chi}\right)$ plane. On the vertical axis, we plot $\lambda_{R}=2 \lambda_{L}$, while on the horizontal axis we plot $m_{\chi}$ in the range $100-200 \mathrm{GeV}$. We keep the mixing angle fixed at $\alpha=\pi / 4$. The mass of the lightest scalar mediator $\tilde{f}_{1}$ is kept at

$$
\mu_{1}=\frac{m_{\tilde{f}_{1}}^{2}}{m_{\chi}^{2}}=1.44
$$

In the region of parameter space plotted, the only constraints come from our HL-LHC study and LZ projections. Current LUX constraints on the anapole moment are too weak to show up in this region, while direct search constraints for the uncolored mediator $\tilde{f}_{1}$ lie below $100 \mathrm{GeV}$. The bluish grey region shows the part of parameter space that will be constrained by mono- $Z$ searches at the HL-LHC, assuming $3000 \mathrm{fb}^{-1}$ of data and 5\% systematic uncertainties. The red region is the region that will be constrained by $\mathrm{LZ}$, assuming the most optimistic performance with one background event in 1000 days of exposure of 5.6 tonne fiducial mass.

We note that lower values of $\mu_{1}$, corresponding to a greater degree of compression between the lightest scalar mediator and the DM, have larger values of the anapole moment from Eq. (6.5). These regions are constrained both by our HL-LHC study and by LZ projections, indeed to a greater extent than is the case in Fig. 8. On the other hand, larger values of $\mu_{1}$ are less suited to our collider search strategy as well as DM direct detection, due to a reduced 


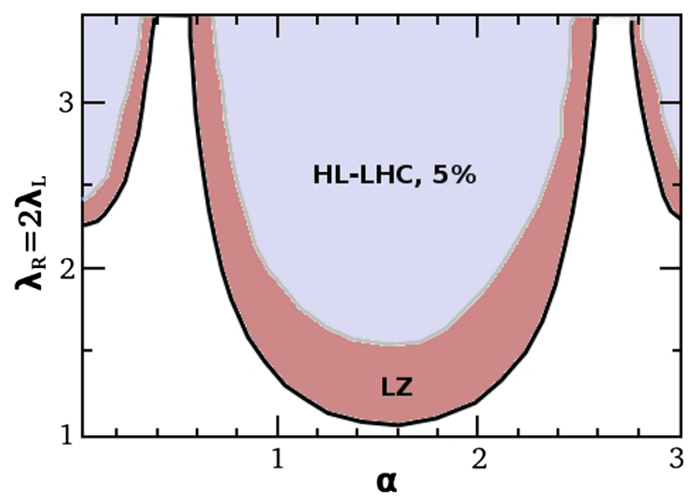

FIG. 9. Constraints on the $(\lambda, \alpha)$ plane: We show constraints on the $\lambda_{R}=2 \lambda_{L}$ versus $\alpha$ plane, keeping $m_{\chi}=200 \mathrm{GeV}$ and $\mu_{1}=m_{\tilde{f}_{1}}^{2} / m_{\chi}^{2}=1.44$. The bluish grey region shows the part of parameter space that will be constrained by mono- $Z$ searches at the HL-LHC, assuming $3000 \mathrm{fb}^{-1}$ of data and $5 \%$ systematic uncertainties. The red region is the region that will be constrained by LZ, assuming the most optimistic performance with one background event in 1000 days of exposure of 5.6 tonne fiducial mass. The LZ projection corresponds to the 1 event contour in Fig. 12.3.6 of [78]. Current LUX limits and direct collider searches for the mediators $\tilde{f}_{1}$ are not able to constrain this part of parameter space.

value of the anapole moment. These regions start becoming constrained by collider searches for the mediators $\tilde{f}$ themselves, as the mass gap between them and the DM increases leading to collider signals with large missing energy.

We show the range of DM masses between 100 and $200 \mathrm{GeV}$. Below $m_{\chi}=100 \mathrm{GeV}$, there are LEP constraints on the uncolored scalar mediators. Above $200 \mathrm{GeV}$, the anapole moment becomes smaller and the constraints become less stringent.

Finally, changing the value of $\alpha$ also changes the anapole moment. We turn to this dependence next.

In Fig. 9, we display the constraints on the $(\lambda, \alpha)$ plane. We keep the mass of DM and the lightest scalar mediator fixed at $m_{\chi}=200 \mathrm{GeV}$ and $\mu_{1}=m_{\tilde{f}_{1}}^{2} / m_{\chi}^{2}=1.44$, respectively. The color scheme is the same as in the previous figure. We see the presence of "blind regions"-regions near $\alpha=\pi / 8,7 \pi / 8$-where the anapole moment becomes highly suppressed, from Eq. (6.4). These regions are difficult to probe using any method that relies on the photon coupling; in [18], however, it was shown that indirect detection can constrain these regions. The effect of changing either $\mu_{1}$ (and hence the light mediator mass) or the mass of the DM has been discussed before, and applies to this figure as well. Lowering $\mu_{1}$ leads to larger values of the anapole moment and stronger constraints on the $(\lambda, \alpha)$ plane, and the "blind regions" get sharpened to values very close to $\alpha=\pi / 8,7 \pi / 8$. Increasing $m_{\chi}$ weakens the collider and direct detection constraints.

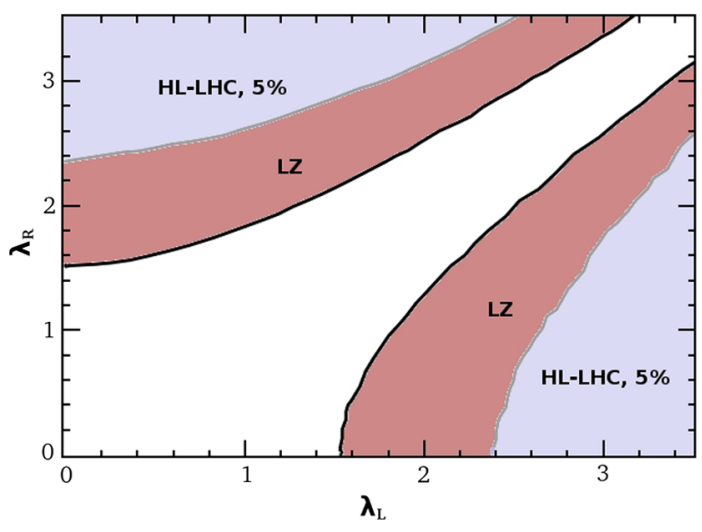

FIG. 10. Constraints on the $\left(\lambda_{R}, \lambda_{L}\right)$ plane: We show constraints on the $\lambda_{R}$ versus $\lambda_{L}$ plane, keeping $m_{\chi}=200 \mathrm{GeV}$, $\mu_{1}=m_{\tilde{f}_{1}}^{2} / m_{\chi}^{2}=1.44$, and $\alpha=\pi / 4$. The bluish grey region shows the part of parameter space that will be constrained by mono- $Z$ searches at the HL-LHC, assuming $3000 \mathrm{fb}^{-1}$ of data and $5 \%$ systematic uncertainties. The red region is the region that will be constrained by LZ, assuming the most optimistic performance with one background event in 1000 days of exposure of 5.6 tonne fiducial mass. The LZ projection corresponds to the 1 event contour in Fig. 12.3.6 of [78]. Current LUX limits and direct collider searches for the mediators $\tilde{f}_{1}$ are not able to constrain this part of parameter space.

The choice of $\lambda_{R}=2 \lambda_{L}$ in Figs. 8 and 9 is taken keeping in mind the supersymmetric limit of this simplified model. In the limit of the MSSM, the dark matter would be the bino, coupling to one generation of light sleptons. In that case, one would have $\left|\lambda_{L}\right|=\sqrt{2} g\left|Y_{L}\right|$ and $\left|\lambda_{R}\right|=\sqrt{2} g\left|Y_{R}\right|$, where $g$ is the electroweak coupling constant, and $\left|Y_{L}\right|=$ $1 / 2$ and $\left|Y_{R}\right|=1$.

Finally, in Fig. 10, we plot our results in the $\left(\lambda_{R}, \lambda_{L}\right)$ plane, keeping $m_{\chi}=200 \mathrm{GeV}, \alpha=\pi / 4$, and $\mu_{1}=1.44$. The color scheme is the same as in the previous figures. The corridor around the region where $\lambda_{R} \sim \lambda_{L}$ constitutes a "blind region" where the anapole moment is attenuated for $\alpha=\pi / 4$. These regions are difficult to probe for methods that rely on the photon coupling. Parts of this region can be explored by indirect detection.

\section{CONCLUSIONS}

In this paper, we have explored the HL-LHC detection prospects for DM that couples to the Standard Model through higher electromagnetic moments, particularly the anapole moment. The study is conducted at the level of EFT, with the aim of calculating the reach in the cutoff scale $\Lambda$. We have conducted our study in the mono- $Z$ channel, taking into account varying levels of systematic uncertainties. We carefully choose kinematic variables that can discriminate between signal and SM background, and select cuts using the Bayesian optimization method implemented in the Python algorithm HYPEROPT. A BDT is then used to classify events into signal and background classes. 
The results of our collider study are shown in Fig. 5. We see that for a very small systematic uncertainty of $1 \%$, the $5 \sigma$ reach in $\Lambda$ is above $1.1 \mathrm{TeV}$ for DM masses in the range 100 $500 \mathrm{GeV}$. Assuming a larger systematic uncertainty of 5\%, a $100 \mathrm{GeV} \mathrm{DM}$ can still be discovered for $\Lambda \approx 1 \mathrm{TeV}$. From $5 \%$ to $10 \%$ and from $10 \%$ to $20 \%$ systematics, the discovery reach in $\Lambda$ decreases by $200 \mathrm{GeV}$ for a given DM mass.

The $5 \sigma$ reach in the cutoff scale $\Lambda$ obtained from our collider study can be mapped onto a reach in the value of the anapole moment $\mathcal{A}$ through Eq. (1.2). The resulting comparative study between the collider reach and the reach from direct detection experiments is shown in Fig. 6. The LUX 2016 results are shown in the brown dash-dot-dotted curve, while the black solid curve shows the projected LZ limit. The green dot-dashed, blue dotted, and red dashed curves show the results obtained from our collider study, with 20\%, 10\%, and 5\% systematic uncertainties and $3000 \mathrm{fb}^{-1}$ of data at the HL-LHC, respectively.

Finally, the EFT analysis is applied to a specific simplified model. We choose a weakly coupled UV completion in which the DM is a Majorana fermion $\chi$ that couples to an uncolored fermion $f$ (with mass $m_{f}$ ) and a pair of charged scalars $\tilde{f}_{L, R}$. At one loop, the DM couples to the photon through an anapole moment interaction. The HL-LHC constraints on the parameter space of this class of models is presented in Figs. 8-10. These constraints are juxtaposed with projected LZ constraints on the parameter space, assuming the most optimistic performance with one background event in 1000 days of exposure of 5.6 tonne fiducial mass.

We make a few comments about the projected timelines of the experiments discussed in this paper. The anticipated timeline for obtaining $3000 \mathrm{fb}^{-1}$ of HL-LHC data is around 2035, according to various documents and talks from the Collaborations (for example, https://project-hl-lhc-industry .web.cern.ch/content/project-schedule). On the other hand, the anticipated timeline for commissioning and for early operations of LZ are estimated to be early 2020 [78]. The timeline for achieving limits $\sim 10^{-48} \mathrm{~cm}^{2}$ on the spin independent (SI) scattering cross section is around 2025, according to Fig. 1.2.2 of the older conceptual design report [73].

The LZ projections shown in the paper assume the most optimistic scenario of one background event in 1000 days of exposure of 5.6 tonne fiducial mass. This corresponds to the 1 event contour in Fig. 12.3.6 of [78]. The $3 \sigma$ and $5 \sigma$ projection contours are more than an order of magnitude less stringent [78]. Assuming these more realistic projections, the resulting constraints on the anapole moment would be a factor $\sim 4-5$ less stringent than the ones depicted in our paper. This would bring the LZ projections more in line with the HL-LHC contour with $10 \%$ systematic errors in our figures. Therefore, depending on the level of systematic errors at the HL-LHC for this class of searches, and the future performance of $\mathrm{LZ}$, the required sensitivity for discovering these candidates may be reached by either experiment.

We thus believe that direct detection and collider searches will be poised to play complementary roles in the discovery of this class of models. However, the bestcase scenario for LZ may start probing this class of models as early as 2025, and in the event of a signal, will guide the HL-LHC in its probe of the relevant parameter space.

\section{ACKNOWLEDGMENTS}

A. A. acknowledges financial support from the Brazilian agencies CNPq, under Project No. 307098/2014-1, and Fundação de Amparo à Pesquisa do Estado de São Paulo (FAPESP), under Project No. 2013/22079-8. A. C. O. S. acknowledges Coordenação de Aperfeiçoamento de Pessoal de Nível Superior (Capes-PDSE-88881.135139/ 2016-01).
[1] C. P. Burgess, M. Pospelov, and T. ter Veldhuis, The minimal model of nonbaryonic dark matter: A singlet scalar, Nucl. Phys. B619, 709 (2001).

[2] F. S. Queiroz and K. Sinha, The poker face of the Majoron dark matter model: LUX to keV line, Phys. Lett. B 735, 69 (2014).

[3] A. Alves, S. Profumo, and F. S. Queiroz, The dark $Z^{\prime}$ portal: Direct, indirect and collider searches, J. High Energy Phys. 04 (2014) 063.

[4] A. Alves and K. Sinha, Searches for dark matter at the LHC: A multivariate analysis in the mono- $Z$ channel, Phys. Rev. D 92, 115013 (2015).

[5] M. Battaglieri et al., US cosmic visions: New ideas in dark matter 2017: Community report, arXiv:1707.04591.
[6] S. Raby and G. West, Experimental consequences and constraints for magninos, Phys. Lett. B 194, 557 (1987).

[7] S. Raby and G. West, Detection of galactic halo magninos via their coherent interaction with heavy nuclei, Phys. Lett. B 200, 547 (1988).

[8] J. Bagnasco, M. Dine, and S. D. Thomas, Detecting technibaryon dark matter, Phys. Lett. B 320, 99 (1994).

[9] K. Sigurdson, M. Doran, A. Kurylov, R. R. Caldwell, and M. Kamionkowski, Dark-matter electric and magnetic dipole moments, Phys. Rev. D 70, 083501 (2004); Erratum, Phys. Rev. D73, 089903(E) (2006).

[10] M. Pospelov and T. ter Veldhuis, Direct and indirect limits on the electromagnetic form-factors of WIMPs, Phys. Lett. B 480, 181 (2000). 
[11] J. F. Fortin and T. M. P. Tait, Collider constraints on dipoleinteracting dark matter, Phys. Rev. D 85, 063506 (2012).

[12] T. Banks, J. F. Fortin, and S. Thomas, Direct detection of dark matter electromagnetic dipole moments, arXiv:1007 .5515 .

[13] O. Antipin, M. Redi, A. Strumia, and E. Vigiani, Accidental composite dark matter, J. High Energy Phys. 07 (2015) 039.

[14] M. I. Gresham and K. M. Zurek, Light dark matter anomalies after LUX, Phys. Rev. D 89, 016017 (2014).

[15] A. L. Fitzpatrick and K. M. Zurek, Dark moments and the DAMA-CoGeNT puzzle, Phys. Rev. D 82, 075004 (2010).

[16] V. Barger, W. Y. Keung, and D. Marfatia, Electromagnetic properties of dark matter: Dipole moments and charge form factor, Phys. Lett. B 696, 74 (2011).

[17] J. Kopp, L. Michaels, and J. Smirnov, Loopy constraints on leptophilic dark matter and internal bremsstrahlung, J. Cosmol. Astropart. Phys. 04 (2014) 022.

[18] P. Sandick, K. Sinha, and F. Teng, Simplified dark matter models with charged mediators: Prospects for direct detection, J. High Energy Phys. 10 (2016) 018.

[19] B. Dutta, T. Ghosh, A. Gurrola, W. Johns, T. Kamon, P. Sheldon, K. Sinha, K. Wang, and S. Wu, Probing compressed sleptons at the LHC using vector boson fusion processes, Phys. Rev. D 91, 055025 (2015).

[20] R. Primulando, E. Salvioni, and Y. Tsai, The dark penguin shines light at colliders, J. High Energy Phys. 07 (2015) 031.

[21] K. Kadota and J. Silk, Constraints on light magnetic dipole dark matter from the ILC and SN 1987A, Phys. Rev. D 89, 103528 (2014).

[22] J. Bergstra, D. Yamins, and D. Cox, in Proceedings of the 30th International Conference on Machine Learning, Atlanta, Georgia, USA, 2013 (JMLR: W\&CP, 2013), Vol. 28.

[23] E. Del Nobile, G. B. Gelmini, P. Gondolo, and J. H. Huh, Direct detection of light anapole and magnetic dipole DM, J. Cosmol. Astropart. Phys. 06 (2014) 002.

[24] T. Li, S. Miao, and Y. F. Zhou, Light mediators in dark matter direct detections, J. Cosmol. Astropart. Phys. 03 (2015) 032.

[25] M. T. Frandsen, F. Kahlhoefer, C. McCabe, S. Sarkar, and K. Schmidt-Hoberg, The unbearable lightness of being: CDMS versus XENON, J. Cosmol. Astropart. Phys. 07 (2013) 023.

[26] P. Gondolo and K. Kadota, Late kinetic decoupling of light magnetic dipole dark matter, J. Cosmol. Astropart. Phys. 06 (2016) 012.

[27] E. Del Nobile, G. B. Gelmini, and S. J. Witte, Prospects for detection of target-dependent annual modulation in direct dark matter searches, J. Cosmol. Astropart. Phys. 02 (2016) 009.

[28] J. F. Cherry, M. T. Frandsen, and I. M. Shoemaker, Direct Detection Phenomenology in Models Where the Products of Dark Matter Annihilation Interact with Nuclei, Phys. Rev. Lett. 114, 231303 (2015).

[29] T. Li, S. Miao, and Y.F. Zhou, Light mediators in dark matter direct detections, J. Cosmol. Astropart. Phys. 03 (2015) 032.

[30] M. I. Gresham and K. M. Zurek, Light dark matter anomalies after LUX, Phys. Rev. D 89, 016017 (2014).
[31] K. Fukushima, C. Kelso, J. Kumar, P. Sandick, and T. Yamamoto, MSSM dark matter and a light slepton sector: The incredible bulk, Phys. Rev. D 90, 095007 (2014).

[32] J. Kumar, P. Sandick, F. Teng, and T. Yamamoto, Gammaray signals from dark matter annihilation via charged mediators, Phys. Rev. D 94, 015022 (2016).

[33] C. Kelso, J. Kumar, P. Sandick, and P. Stengel, Charged mediators in dark matter scattering with nuclei and the strangeness content of nucleons, Phys. Rev. D 91, 055028 (2015).

[34] A. Ibarra and S. Wild, Dirac dark matter with a charged mediator: A comprehensive one-loop analysis of the direct detection phenomenology, J. Cosmol. Astropart. Phys. 05 (2015) 047.

[35] M. Garny, A. Ibarra, and S. Vogl, Signatures of Majorana dark matter with $t$-channel mediators, Int. J. Mod. Phys. D 24, 1530019 (2015).

[36] P. Sandick, K. Sinha, and T. Yamamoto, Black holes, dark matter spikes, and constraints on simplified models with t-channel mediators, arXiv:1701.00067.

[37] S. P. Liew, M. Papucci, A. Vichi, and K. M. Zurek, Mono-X versus direct searches: Simplified models for dark matter at the LHC, J. High Energy Phys. 06 (2017) 082.

[38] A. G. Delannoy et al., Probing Dark Matter at the LHC using Vector Boson Fusion Processes, Phys. Rev. Lett. 111, 061801 (2013).

[39] C. M. Ho and R. J. Scherrer, Anapole dark matter, Phys. Lett. B 722, 341 (2013).

[40] S. Matsumoto, S. Mukhopadhyay, and Y. L. S. Tsai, Singlet Majorana fermion dark matter: A comprehensive analysis in effective field theory, J. High Energy Phys. 10 (2014) 155.

[41] L. G. Cabral-Rosetti, M. Mondragn, and E. Reyes-Prez, Anapole moment of the lightest neutralino in the cMSSM, Nucl. Phys. B907, 1 (2016).

[42] D. C. Latimer, Anapole dark matter annihilation into photons, Phys. Rev. D 95, 095023 (2017).

[43] Y. Gao, C. M. Ho, and R. J. Scherrer, Anapole Dark Matter at the LHC, Phys. Rev. D 89, 045006 (2014).

[44] A. Ibarra, C. E. Yaguna, and O. Zapata, Direct detection of fermion dark matter in the radiative seesaw model, Phys. Rev. D 93, 035012 (2016).

[45] G. Kane, K. Sinha, and S. Watson, Cosmological moduli and the post-inflationary universe: A critical review, Int. J. Mod. Phys. D 24, 1530022 (2015).

[46] B. Dutta, L. Leblond, and K. Sinha, Mirage in the sky: Nonthermal dark matter, gravitino problem, and cosmic ray anomalies, Phys. Rev. D 80, 035014 (2009).

[47] B. Geytenbeek, S. Rao, P. Scott, A. Serenelli, A. C. Vincent, M. White, and A. G. Williams, Effect of electromagnetic dipole dark matter on energy transport in the solar interior, J. Cosmol. Astropart. Phys. 03 (2017) 029.

[48] J. Goodman, M. Ibe, A. Rajaraman, W. Shepherd, T. M. P. Tait, and H. B. Yu, Constraints on light Majorana dark matter from colliders, Phys. Lett. B 695, 185 (2011).

[49] J. Goodman, M. Ibe, A. Rajaraman, W. Shepherd, T. M. P. Tait, and H. B. Yu, Constraints on dark matter from colliders, Phys. Rev. D 82, 116010 (2010). 
[50] N. F. Bell, J. B. Dent, A. J. Galea, T. D. Jacques, L. M. Krauss, and T. J. Weiler, Searching for dark matter at the LHC with a Mono-Z, Phys. Rev. D 86, 096011 (2012).

[51] Y. Gershtein, F. Petriello, S. Quackenbush, and K. M. Zurek, Discovering hidden sectors with mono-photon $Z^{\prime}$ o searches, Phys. Rev. D 78, 095002 (2008).

[52] F. J. Petriello, S. Quackenbush, and K. M. Zurek, The invisible $Z^{\prime}$ at the CERN LHC, Phys. Rev. D 77, 115020 (2008).

[53] L. M. Carpenter, A. Nelson, C. Shimmin, T. M. P. Tait, and D. Whiteson, Collider searches for dark matter in events with a $Z$ boson and missing energy, Phys. Rev. D 87, 074005 (2013).

[54] Y. Bai and T. M. P. Tait, Searches with mono-leptons, Phys. Lett. B 723, 384 (2013).

[55] A. A. Petrov and W. Shepherd, Searching for dark matter at LHC with mono-Higgs production, Phys. Lett. B 730, 178 (2014).

[56] Y. Bai, P. J. Fox, and R. Harnik, The tevatron at the frontier of dark matter direct detection, J. High Energy Phys. 12 (2010) 048.

[57] A. Alves, T. Ghosh, and K. Sinha, Can we discover double Higgs production at the LHC?, Phys. Rev. D 96, 035022 (2017).

[58] A. Alloul, N. D. Christensen, C. Degrande, C. Duhr, and B. Fuks, FeynRules 2.0 - A complete toolbox for tree-level phenomenology, Comput. Phys. Commun. 185, 2250 (2014).

[59] J. Alwall, M. Herquet, F. Maltoni, O. Mattelaer, and T. Stelzer, MadGraph 5: Going beyond, J. High Energy Phys. 06 (2011) 128.

[60] M. L. Mangano, M. Moretti, F. Piccinini, and M. Treccani, Matching matrix elements and shower evolution for topquark production in hadronic collisions, J. High Energy Phys. 01 (2007) 013.

[61] T. Sjostrand, S. Mrenna, and P.Z. Skands, PYTHIA 6.4 physics and manual, J. High Energy Phys. 05 (2006) 026.

[62] J. de Favereau et al. (DELPHES 3 Collaboration), DELPHES 3, A modular framework for fast simulation of a generic collider experiment, J. High Energy Phys. 402 (2014) 057.

[63] M. Cacciari, G. P. Salam, and G. Soyez, FastJet user manual, Eur. Phys. J. C 72, 1896 (2012).

[64] G. Aad et al. (ATLAS Collaboration), Measurement of ZZ production in $p p$ collisions at $\sqrt{s}=7 \mathrm{TeV}$ and limits on anomalous $Z Z Z$ and $Z Z \gamma$ couplings with the ATLAS detector, J. High Energy Phys. 03 (2013) 128.
[65] L. Edelhuser, M. Krämer, and J. Sonneveld, Simplified models for same-spin new physics scenarios, J. High Energy Phys. 04 (2015) 146.

[66] A. J. Barr, Measuring slepton spin at the LHC, J. High Energy Phys. 02 (2006) 042.

[67] A. Alves and O. Eboli, Unravelling the sbottom spin at the CERN LHC, Phys. Rev. D 75, 115013 (2007).

[68] D. R. Tovey, On measuring the masses of pair-produced semi-invisibly decaying particles at hadron colliders, J. High Energy Phys. 04 (2008) 034.

[69] Chen, Tianqi, and Guestrin, in Proceedings of the 22nd ACM SIGKDD International Conference on Knowledge Discovery and Data Mining, San Francisco, CA, USA (2016).

[70] P. Baldi, K. Cranmer, T. Faucett, P. Sadowski, and D. Whiteson, Parameterized neural networks for high-energy physics, Eur. Phys. J. C 76, 235 (2016).

[71] C. M. Ho and R. J. Scherrer, Sterile neutrinos and light dark matter save each other, Phys. Rev. D 87, 065016 (2013).

[72] D. S. Akerib et al. (LUX Collaboration), Results from a Search for Dark Matter in the Complete LUX Exposure, Phys. Rev. Lett. 118, 021303 (2017).

[73] D. S. Akerib et al. (LZ Collaboration), LUX-ZEPLIN (LZ) conceptual design report, arXiv:1509.02910.

[74] D. S. Akerib et al. (LUX Collaboration), Improved Limits on Scattering of Weakly Interacting Massive Particles from Reanalysis of 2013 LUX Data, Phys. Rev. Lett. 116, 161301 (2016).

[75] O. Buchmueller, M. J. Dolan, and C. McCabe, Beyond effective field theory for dark matter searches at the LHC, J. High Energy Phys. 01 (2014) 025.

[76] J. Goodman, M. Ibe, A. Rajaraman, W. Shepherd, T. M. P. Tait, and H. B. Yu, Constraints on dark matter from colliders, Phys. Rev. D 82, 116010 (2010).

[77] L. M. Carpenter, A. Nelson, C. Shimmin, T. M. P. Tait, and D. Whiteson, Collider searches for dark matter in events with a $Z$ boson and missing energy, Phys. Rev. D 87, 074005 (2013).

[78] B. J. Mount et al., LUX-ZEPLIN (LZ) technical design report, arXiv:1703.09144.

[79] M. Aaboud et al. (ATLAS Collaboration), Search for dark matter and other new phenomena in events with an energetic jet and large missing transverse momentum using the ATLAS detector, J. High Energy Phys. 01 (2018) 126.

[80] M. Aaboud et al. (ATLAS Collaboration), Performance of the ATLAS trigger system in 2015, Eur. Phys. J. C 77, 317 (2017).

[81] K. Fukushima and J. Kumar, Dipole moment bounds on dark matter annihilation, Phys. Rev. D 88, 056017 (2013). 